\title{
Binding Browsing and Reading Activities in a 3D Digital Library
}

\author{
Pierre Cubaud, Pascal Stokowski, Alexandre Topol \\ Centre d'Etudes et de Recherche en Informatique (CEDRIC) \\ Conservatoire National des Arts et Métiers, Paris, France \\ \{cubaud, stokow_p, topol\}@cnam.fr http://cedric.cnam.fr/mihm/
}

\section{ABSTRACT}

Browsing through digitalized books collections and reading activities are separated in most present WWW-based user's interfaces of digital libraries. This context break induces longer apprenticeship and navigation time within the interface. We study in this paper how 3D interaction metaphors provide a continuous navigation space for these two tasks.

\section{Keywords \\ Digital Library, 3D interaction, Virtual Reality \\ INTRODUCTION}

Studies like [1] have shown that 3D interfaces can be more powerful than classical 2D interfaces managing overlapping windows. This efficiency can be reached when proposing the right objects and navigation/interaction metaphors. The most often used approach is to take the ones from the real physical space. When creating an interface in which it is important to have both the real world's behaviors and look like in a game or a simulator, this virtual reality approach makes sense. But, in other domains, a 3D interface should pass over those limitations. For 3D digital libraries, we think that there is no reason to reproduce faithfully a real library building as it is done in $[4,5]$. A positive point in this "real" to "virtual" mapping is to ease the retrieving of books for users who are familiar with the real library. However, for new users that are mostly the ones we deal with on the internet, this approach does not help. Moreover, walking around bookshelves is not easy for 3D novice users. We believe it is more appropriate to provide an abstract navigation and offer services like reordering books in space depending on search criterions [2].

Different tools are required to find, access, read and work on books facsimiles: typically, a search engine helps to find books and a reading tool to work with the chosen ones. In a virtually, reconstituted, library, the bookshelves and the reading desk are tightly and naturally connected. This results however in an increased time spent in navigating through the 3D space. On the other hand, abstract tools such as the one we have implemented separately in $[2,3]$ are more difficult to integrate within a common framework.

\section{DESIGN CHOICES}

Two options are possible for binding browsing and reading activities. The first one is to mix their content and the second is to separate them in space and use a 3D metaphor to switch from one to the other. Whatever solution we choose, the first important point is to propose a common set of metaphors so that both tools can be integrated smoothly without disorienting the user. The collection is however the main element of the library since the user's interest and work depends upon it. Another approach is a documentcentered interface like the Web Forager [1] in which documents are the main elements and tools are produced at demand as secondary pop-up resources.

We provide a constrained browsing within the collection in order to guide beginners, but it should give enough freedom to expert users. The user can also open a book to take a quick glance at its content before selecting it. Some interactions will therefore be necessary here. In the reading interface users can not move but only interact with objects. Hence, interactions in the reading interface are almost a subset of those allowed within the bookshelves. To link one tool with the other it as to be done by a continuous movement. The goal is to avoid using widgets like buttons and to keep as stated previously the same interaction metaphors. We now describe some sketches of such navigation metaphors.

\section{INTEGRATION METAPHORS}

In the three integration metaphors proposed, the basket (shown in the three figures) is the only common tool, always visible and in the foreground. It serves as a link

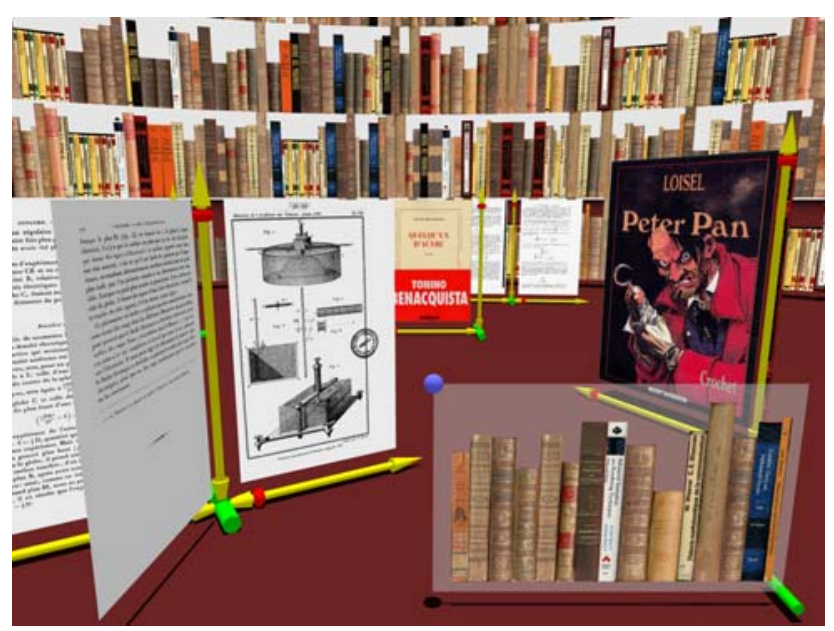

Figure 1 : The cockpit metaphor 
between both interfaces. It is used to group the pre-selected books chosen in the collection interface, to access them in the reading one and also to save the user's reading session.

\section{The cockpit metaphor}

The scene's background is made of the books collection (fig. 1). The basket and the opened books are in the foreground. They are always visible and are not affected by the user's navigation. As a pilot flies over a landscape while seeing some data in his helmet, in this interface the user navigates through the collection and sees the books. The main problems are the surcharged screen and the difficulty to see and access the collection behind opened books. Another main issue is to keep a maximum space for both tools. Hence, two separated areas in a same interface seems a good choice.

\section{Horizontally structured workspace}

The space is separated in two horizontal zones (fig. 2). The user can switch from one to the other by rotating around the $\mathrm{Y}$ axis. In the first zone, the collection is rendered and the user can move the point of view. In the second one, the books are always displayed on the ground. Hence, when the user moves in the collection zone, the ground and books also move behind to simulate their immobility.

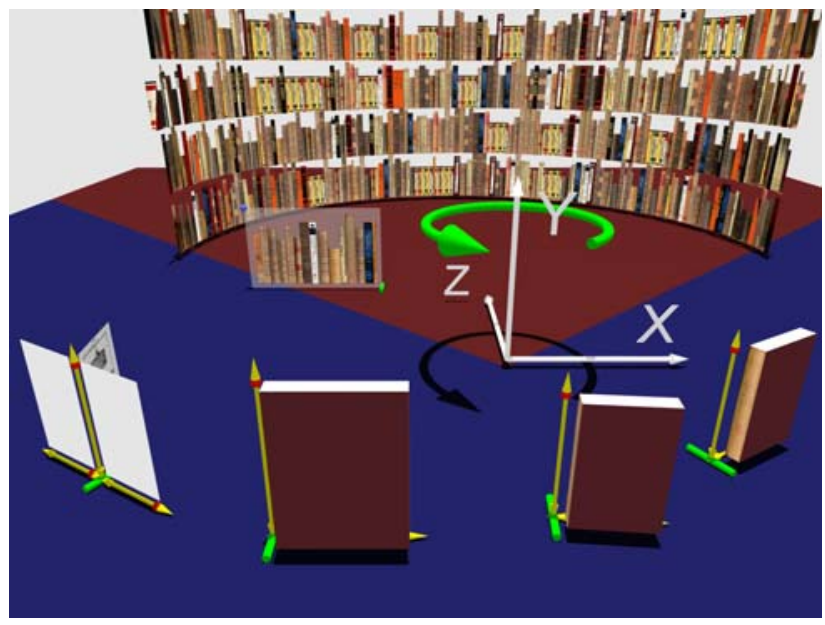

Figure 2 : Horizontally structured workspace

With this metaphor, the user can also create several reading environments depending on their orientation within the reading area and the view angle of the camera.

\section{Vertically structured workspace}

There is a main problem with the previous metaphor : after moving in the collection zone, some previously opened books in the reading zone can be hidden. Moreover, half of the space - under the ground - is not used. To avoid these limitations, we can split the 3D space in two vertical zones instead of two horizontal ones. Moving the point of view in the collection zone will not affect the view in the reading zone. To switch from one zone to the other, a rotation around the $\mathrm{Z}$ axis can be used. In the reading zone, the user can still create several reading environments by rotating around the $\mathrm{Y}$ axis. The fig. 3 shows the two zones separated by the ground. Of course, the user is immersed in

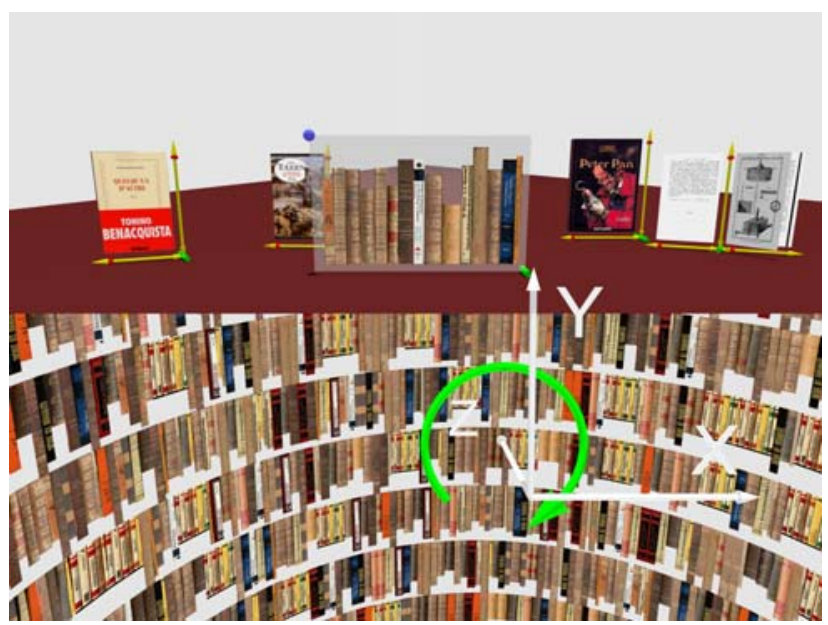

Figure 3 : Vertically structured workspace

the scene. These far views are only given to understand how the two interfaces work.

\section{CONCLUSION - FUTURE WORK}

The metaphors described previously are not implemented in a real time system yet but are only sketched with scripted POV files (available on our website). Since creating an online library with VRML appeared to be difficult [3], we shall first build either a stand-alone local application using a 3D API or an online application using a visual tool like Virtools Dev or Cult3D. We shall then study different representations for the display of the collection. We could use a cylindrical shaped collection like in [2] but also other metaphors like semantic maps, cone trees, ... The idea is to display the collection of a particular library with a default metaphor chosen by the librarian who knows which one is the more adapted. A default sort method for books would also be chosen when entering the library for the first time. The user would however be allowed to change those settings to some other he is more familiar or efficient with.

\section{REFERENCES}

1. S. Card, G. Robertson, W. York. The WebBook and the Web Forager: An Information Workspace for the World-Wide-Web. In Proc of ACM CHI'96. Vancouver, April 1996.

2. P. Cubaud, C. Thiria, A. Topol. Experimenting a 3D Interface for the access to a Digital Library. In Proc of ACM DL'98. Pittsburg, USA, June 1998.

3. P. Cubaud, A. Topol. A VRML-based user interface for an online digitalized antiquarian collection. In Proc of ACM Web3D'2001, Paderdorn, Germany, Feb. 2001.

4. E. Fox, N. Kipp et al. Networked Digital Library of Theses and Dissertations : An International Effort Unlocking University Resources. D-lib magazine, Sept. 1997.

5. P. Plénacoste, E. Lecolinet, S. Pook, C. Dumas, J-D. Fekete, Zoomable and 3D Representations for Digital Libraries, In Proc of IHM-HCI 2001, Lille, Sept. 2001 